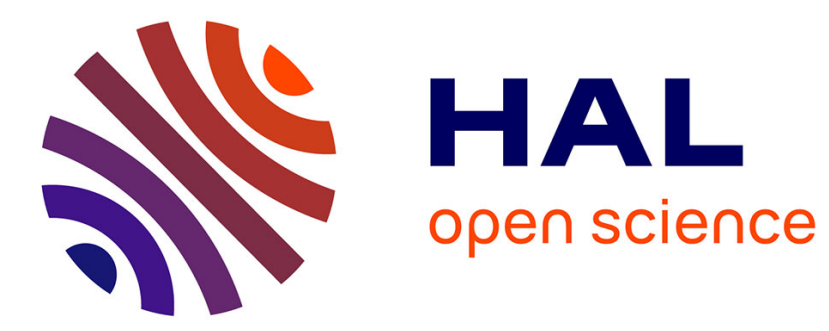

\title{
Statistiques et normes : jalons pour une rencontre interdisciplinaire
}

Olivier Leclerc

\section{To cite this version:}

Olivier Leclerc. Statistiques et normes : jalons pour une rencontre interdisciplinaire. Cahiers, Droit, Sciences \& Technologies, 2014, 4, pp.37-44. 10.4000/cdst.298 . halshs-01085319

\section{HAL Id: halshs-01085319 \\ https://shs.hal.science/halshs-01085319}

Submitted on 1 Oct 2015

HAL is a multi-disciplinary open access archive for the deposit and dissemination of scientific research documents, whether they are published or not. The documents may come from teaching and research institutions in France or abroad, or from public or private research centers.
L'archive ouverte pluridisciplinaire HAL, est destinée au dépôt et à la diffusion de documents scientifiques de niveau recherche, publiés ou non, émanant des établissements d'enseignement et de recherche français ou étrangers, des laboratoires publics ou privés. 


\section{Statistiques et normes : jalons pour une rencontre interdisciplinaire Olivier LECLERC*}

paru in Cahiers Droit, Sciences \& Technologies, n 4, 2014, , pp. 37-44.

Résumé : A partir du texte de Robert Salais «La donnée n'est pas un donné. Pour une analyse critique de l'évaluation chiffrée de la performance » (2010), l'article engage la discussion afin d'explorer les voies d'un échange interdisciplinaire sur les rapports entre normes et statistiques. L'article s'attache à la construction de l'objectivité, aux usages des statistiques dans la décision publique, aux usages des statistiques dans les sciences sociales.

Abstract : Statistics and Norms: Towards an Interdisciplinary Dialogue : The paper builds on Robert Salais' paper "Statistics do not stand alone: critical analysis of the quantitative assessment of public sector performance » (2010). It aims at engaging an interdisciplinary dialogue on the relationships between norms and statistics. The paper focuses on the construction of objectivity, the role of statistics into decision-making, the place of statistics in the social sciences.

Je voudrais commencer cette discussion par une remarque générale, destinée à situer précisément le type d’objets que nous considérons aujourd'hui. Ainsi que l'a fait observer Alain Desrosières dans l'un de ses textes, les chiffres diffusés par les services statistiques publics proviennent de deux sources bien différentes : « les enquêtes directes (recensements, sondages...) et les registres administratifs (fichiers de gestion, répertoires...) » ${ }^{1}$. Les objectifs poursuivis par ces dispositifs de recueil d'information statistique diffèrent profondément : alors que les enquêtes visent à décrire certains aspects de la société ${ }^{2}$, les registres sont conçus à des fins de gestion et reflètent plutôt les rouages de l'État et des institutions qui les mettent en place. C’est de ces derniers qu'il est question au cours de cette matinée consacrée à la

1 * Chargé de recherche CNRS, Université de Lyon, CERCRID (UMR 5137), Université Jean Monnet, Saint-Étienne. Contact : olivier.leclerc@univ-st-etienne.fr A. DESROSIÈREs, « Décrire l’État ou explorer la société : les deux sources de la statistique publique », Genèses, n58, 2005, pp. 4-27.

${ }^{2}$ Selon des clés d'interprétation et en réponse à des interrogations données. 
formation du droit de la santé et à la critique de la raison statistique ${ }^{3}$. A l'image des statistiques judiciaires ${ }^{4}$, les statistiques dont nous parlons ne sont pas le fruit de grandes enquêtes mais des sous-produits de gestion de l'activité médicale. Il existe ainsi une liaison intime entre statistiques et activité médicale, qui s’appuie sur la mise en place de systèmes de gestion permettant la collecte d'informations (logiciels de gestion des cabinets médicaux, circulation de flux d’informations entre les médecins et les organismes de sécurité sociale...).

En réaction à l’intervention de Robert Salais, et plus généralement au texte « La donnée n’est pas un donné $»^{5}$ qui nous sert aujourd'hui de point d'accroche pour engager la discussion, je vais mettre l'accent sur trois séries de remarques. Pour chacune d'entre elles, je m'efforcerai de souligner les objets et les questionnements qui ouvrent des espaces d'échange entre économistes, sociologues et juristes. Je ferai part également, lorsque ce sera nécessaire, de quelques interrogations ou sujets de perplexité.

\section{Objectivité statistique et conventions de mesure}

Une première série d'observations porte sur l'objectivité. L'un des points importants soulevés par Robert Salais est que les statistiques ne saisissent pas directement le réel ; elles sont le produit de conventions de mesure, de mises en forme et, partant, de choix, rarement explicités. Cette idée peut être généralisée pour dire que la nature, comme le social, ne parlent jamais directement : ils sont toujours saisis au travers d'instruments de connaissance - que certains appellent des « inscripteurs » ou «systèmes d'inscription ${ }^{6}-$. Or, ce que montre Robert Salais, c'est qu'il convient de dénaturaliser les instruments d'inscription et de prêter attention à leur construction, aux choix qu'ils traduisent, à ce qu'ils permettent de « voir » comme à ce qu'ils empêchent de voir. C'est ainsi que les indicateurs sont décrits par Robert Salais comme des «représentations cognitives des situations $»^{7}$. Alain Desrosières l'a aussi

${ }^{3}$ Voir les contributions de A.-S. Ginon et I. Vacarie dans ce dossier.

${ }^{4}$ E. SERVERIN, « De la statistique judiciaire civile et de ses usages », Revue internationale de droit économique, n², 1999, pp. 281-294, spéc. pp. 285 et s. V. également Ch. BEROuJON et S. BRUXELlES, "Règles juridiques, catégories statistiques et actions sociales ", Droit et société, n²5, 1993, pp. 369-394.

${ }^{5}$ R. SALAIS, « La donnée n'est pas un donné. Pour une analyse critique de l'évaluation chiffrée de la performance », Revue française d'administration publique, $\mathrm{n}^{\circ} 135$, 2010, pp. 497-515.

${ }^{6}$ B. LATOUR et S. WOOLGAR, La vie de laboratoire. La production des faits scientifiques, trad. M. Biezunski, Paris, La découverte, [1979] 1988, p. 42.

${ }^{7} \mathrm{R}$. SALAIS, « La donnée n'est pas un donné. Pour une analyse critique de l'évaluation chiffrée de la performance », loc. cit., p. 514. 
très bien montré dans La politique des grands nombres ${ }^{8}$ : la quantification est un mode de connaissance dont il est possible de retracer l'émergence et de suivre les trajectoires. Alain Desrosières parle ailleurs de « mise en forme cognitive $»^{9}$.

Cette position ouvre, à mon sens, au moins deux voies d’échange interdisciplinaire. Elle est aussi source d'une interrogation.

a. Afin d'identifier un premier espace de dialogue, il est utile de souligner que cette idée, selon laquelle l'outillage statistique construit une forme d'objectivité chiffrée - dit autrement, que «l'objectivité des données quantifiées [résulte] de conventions de mesure » ${ }^{10}$-, est présente dans l'histoire sociale et culturelle des sciences. Lorraine Daston et Peter Galison ${ }^{11}$ montrent clairement que la vertu savante de l'objectivité a une histoire. Menant une analyse approfondie des formes d'exposition retenues dans les atlas naturalistes depuis le début du XVIII ${ }^{\text {ème }}$ siècle, ces auteurs retracent une histoire des mises en forme de l'objectivité. Il en ressort qu'une représentation de la nature ou du social n'est reconnue comme objective que si elle suit des conventions de forme, elles-mêmes changeantes. Ainsi, dans un article sur « la formation du chômage dans les années $1930 »^{12}$, Robert Salais montre que, si le chômage est un phénomène lié à l'industrialisation et à l'urbanisation, la catégorie de chômeur a aussi émergé grâce à des dispositifs d'enregistrement, des formulaires, des catégories qui permettaient de la saisir. Pour faire advenir - et compter - la position de " chômeur », il faut disposer d'un appareil statistique de recueil d'informations ainsi que de catégories juridiques appropriées. Ce n’est, ainsi, pas le chômage qui s’impose au droit comme un phénomène à encadrer ; la notion est elle-même le fruit d'un travail de mise en ordre du social auquel participe également le droit, notamment au travers de la création de catégories juridiques comme le rapport salarial, la subordination juridique. En somme, les logiques de dénombrement supposent l'établissement d'instruments de mesure et de catégories signifiantes, dont il est possible de retracer la genèse et l'évolution.

${ }^{8} \mathrm{~A}$. DesRosières, La politique des grands nombres. Histoire de la raison statistique, Paris, La découverte, coll. « Textes à l'appui », 1993.

${ }^{9} \mathrm{~A}$. Desrosières, Pour une sociologie historique de la quantification. I. L'argument statistique, Paris, Presses de l'Ecole des Mines, coll. « Sciences sociales », 2008, p. 123.

${ }^{10} \mathrm{R}$. SALAIS, « La donnée n'est pas un donné. Pour une analyse critique de l'évaluation chiffrée de la performance », loc. cit., p. 502.

${ }^{11}$ L. DASTON et P. GaLison, Objectivity, New York, Zone Books, 2007.

${ }^{12} \mathrm{R}$. SALAIS, «La formation du chômage dans les années 1930 ", Economie et statistique, $\mathrm{n}^{\circ} 155,1983$, pp. 15-28. 
L’attention prêtée à cette histoire ouvre un premier terrain de dialogue entre sociologues, économistes, historiens et juristes, portant sur le droit des dispositifs d'enregistrement. Comment le droit met-il en place les instruments destinés à saisir le social ? Ce questionnement conduit à s’intéresser à des outils bien trop peu regardés comme les nomenclatures, les classifications, les barèmes, les formulaires de saisie administratifs, les questionnaires, les cartes, les architectures logicielles des systèmes d'information.

b. Le dialogue interdisciplinaire peut, me semble-t-il, se poursuivre sur un terrain connexe, en prenant au sérieux la question posée par Robert Salais de savoir ce qui fait que l'on a confiance dans les statistiques. D’où ces dernières tirent-elles leur force de conviction ? D’où vient cette impression qu'elles donnent accès au réel ? Chaïm Perelman et Lucie Olbrechts-Tyteca rangent les arguments statistiques et probabilistes dans la catégorie des « arguments quasi logiques », qui «prétendent à une certaine forme de conviction, dans la mesure où ils se présentent comme comparables à des raisonnements formels, logiques et mathématiques $»^{13}$. C'est là un deuxième terrain de discussion : celui de la théorie de l'argumentation et des usages des arguments formels - et plus généralement du chiffre - dans les politiques publiques et dans le procès. Sur ces terrains, des travaux d'histoire, de sociologie, de science politique voisinent avec des travaux de juristes, sans que l'articulation entre eux soit pleinement satisfaisante.

c. Mais au-delà des espaces de dialogue ainsi identifiés, demeure une interrogation. En effet, dans son texte «La donnée n’est pas un donné », Robert Salais ne traite pas seulement l’objectivité par référence aux supports matériels qui permettent de l'établir ; il intègre aussi à l'objectivité une dimension intersubjective: un processus social de connaissances n'est objectif qu'à la condition qu'il existe un certain accord sur les données produites. Comme l'écrit Robert Salais, «pour satisfaire à ces deux conditions de justesse et de justice, autrement dit pour être objectif, un processus social de connaissances doit donc être inséparable de formes démocratiques de délibération publique et du débat contradictoire ${ }^{14}$. A défaut, risque de survenir un divorce entre les chiffres et la réalité vécue par les citoyens ${ }^{15}$. Il

${ }^{13}$ C. Perelman et L. Olbrechts-Tyteca, Traité de l'argumentation, 6 e éd., Bruxelles, Éditions de l'université de Bruxelles, 2008, p. 259.

${ }^{14} \mathrm{R}$. SALAIS, « La donnée n'est pas un donné. Pour une analyse critique de l'évaluation chiffrée de la performance », loc. cit., p. 504 (souligné par nous).

${ }^{15}$ Sur cet écart entre les catégories statistiques et la représentation de soi des personnes visées 
existe un rapport à construire, dans une démocratie, entre connaissance et action publique. Dit autrement, la constitution d'un mode objectif de connaissance du social ne passe pas seulement par l'élaboration de dispositifs matériels de recueil et de mise en forme d'informations ; il suppose aussi un accord minimal des personnes saisies par le dispositif sur la représentation que ce dernier donne d'elles. Que la légitimité, ou plus simplement l'acceptation (ou, plus modestement encore, l'absence de contestation), des outils de mesure statistique et des indicateurs suppose effectivement la mise en délibération des classes élaborées et des identités qu'elles équipent, cela me semble parfaitement recevable. En revanche, rattacher cette reconnaissance intersubjective des identités au registre de l'objectivité constitue un déplacement supplémentaire qui appelle, à mes yeux, une discussion plus approfondie.

\section{Dynamique des usages des statistiques dans la décision publique}

Une deuxième observation a trait aux évolutions des usages des statistiques qu’il est possible de déceler dans les politiques publiques. A ce sujet, Robert Salais signale deux modèles de la décision publique $^{16}$. En simplifiant, dans le premier, qui est qualifié de "classique », la constitution d'une base de connaissance relative à son objet est préalable à la décision publique. Cela implique de rechercher un certain accord sur les données produites, leur exactitude et leur conformité à des principes de justice. Par opposition, dans un second modèle, dont le New public management est l'illustration phare, le pilotage s'opère par la seule recherche de la plus grande performance, de sorte que les tableaux de bord sont élaborés de manière non-démocratique. En cela, le New public management est pensé comme érigeant l'efficacité en une fin en soi, et non comme un moyen pour atteindre un objectif collectivement délibéré.

La désignation de ces modèles de la décision publique comme étant l'un « classique » et l'autre « nouveau » laisse inévitablement penser qu'un passage s'est produit historiquement de l'un à l'autre. Pourtant à lire attentivement le texte, cette séquence chronologique par le recueil de données, v. aussi J. AfFICHARD, « La fonction de l'enquête statistique dans l'évaluation du dispositif de formation des jeunes de 16 à 18 ans ", Formation Emploi, n 9 , 1985, pp. 66-74.

${ }^{16} \mathrm{R}$. SALAIS, « La donnée n'est pas un donné. Pour une analyse critique de l'évaluation chiffrée de la performance », loc. cit., p. 504 et s. V. aussi R. SALAIS, "Usages et mésusages de l'argument statistique: le pilotage des politiques publiques par la performance ", Revue française des affaires sociales, $\mathrm{n}^{\circ}$ 1, 2010, spéc. p. 133 et s. 
n’apparaît pas si nette, faute de parvenir à identifier avec fermeté les inflexions remarquables qui permettent de diagnostiquer un changement de modèle. Il est ainsi malaisé de déterminer si ces modèles se sont succédés dans le temps ou s'il s’agit d’idéal-types des modes d'usage des statistiques dans la décision publique. Deux exemples illustrent cette difficulté.

Un premier exemple se rattache à la normativité cachée que charrient les indicateurs dans le modèle du New public management. En effet, dans le même temps que les indicateurs sont porteurs de choix d’un monde désirable, cette normativité est incorporée dans une matière hautement technique pour le profane (choix des indicateurs, de leurs modalités de calcul, des sources statistiques utilisées, des classifications) $)^{17}$. Cette complexité, qui rend l'appareil statistique hermétique aux citoyens non-spécialistes, conduit à ce que la normativité qui lui est attachée leur soit dissimulée et qu'elle soit soustraite à leur jugement. Cette idée ne peut manquer d'emporter l'adhésion. Mais est-il exact que cette obscurité des statistiques pour les citoyens n'existe pas dans le modèle « classique » de décision publique ? On peut en douter si l'on considère les appareils statistiques de mesure de l'activité comme la comptabilité nationale, la mesure du Produit intérieur brut ou la nomenclature des professions et catégories socioprofessionnelles $^{18}$, qui pourraient être rattachés au premier modèle décrit par Robert Salais.

La même difficulté se rencontre lorsque Robert Salais indique que les sciences sociales doivent s'intéresser aux conditions dans lesquelles les citoyens participent ou non, par quels

\footnotetext{
${ }^{17} \mathrm{R}$. SALAIS, « La donnée n'est pas un donné. Pour une analyse critique de l'évaluation chiffrée de la performance ", loc. cit., p. 512 ; R. SALAIS, "Indicateurs et conduite des politiques publiques : où sont les problèmes ? ", Informations sociales, $n^{\circ}$ 167, 2011, pp. 60-70. Voir également A. DesRosiÈres et L. THÉVENOT, « Les mots et les chiffres : les nomenclatures socioprofessionnelles », Economie et Statistique, n 110, 1979, pp. 49-65 ; L. THÉVENOT, « L'économie du codage social », in Critiques de l'économie politique, n²3-24, 1983, pp. 188-222, spéc. p. 206 et s.

${ }^{18}$ Sur ces aspects, voir les monographies de F. FOURQUET, Les comptes de la puissance. Histoire de la comptabilité nationale et du plan, Éditions Encres, 1980 ; M. VOLLE, Histoire de la statistique industrielle, Paris, Economica, 1982 ; A. DEsRosières et L. THÉVENOT, Les catégories socioprofessionnelles, Paris, La découverte, coll. « Repères », 2002. V. aussi A. DesrosiÈres, A. GOY, L. ThÉVEnOt, "L'identité sociale dans le travail statistique », Economie et Statistique, $\mathrm{n}^{\circ}$ 152, 1983, pp. 55-81. Dans l'espace anglophone, v. aussi Donald MacKenzIE, Statistics in Britain, 1865-1930: The Social Construction of Scientific Knowledge, Edinburgh University Press, 1984 ; Th. M. PORTER, Trust in Numbers. The Pursuit of Objectivity in Science and Public Life, Princeton University Press, 1995 ; M. Poovey, A History of the Modern Fact. Problems of Knowledge in the Sciences of Wealth and Society, The University of Chicago Press, 1998.
} 
moyens et à quel niveau, à l'élaboration des cadres cognitifs. L'enjeu est de rendre ces derniers adéquats à ce qu'ils considèrent être un traitement juste des problèmes qu'ils rencontrent ${ }^{19}$. Là encore, le propos convainc sans difficulté. Mais la situation est-elle véritablement différente dans le modèle « classique » et dans le modèle « nouveau » ? On peut douter que l'association des citoyens ou, plus simplement, la mise en délibération publique de l'élaboration des appareillages statistiques, assurément déficiente dans le New public management, ait été meilleure par le passé. Comme le montre James Scott, il existe une asymétrie fondamentale dans les moyens dont disposent les États et les citoyens pour « voir » le monde ; tous les acteurs ne disposent pas des mêmes moyens que l'État pour rendre le monde lisible ${ }^{20}$.

\section{Statistique sociale et normativité}

Une troisième série d'observations porte sur les usages des statistiques dans les sciences sociales, plus particulièrement sur la statistique sociale, qui prétend saisir le social par la statistique. Ce détour, plus historique, m'apparaît utile pour caractériser précisément la cible visée par Robert Salais. Il me semble, en effet, que la position de Robert Salais s’inscrit, à certains égards, dans le droit fil d'auteurs comme Maurice Halbwachs ou François Simiand, mais qu'elle s'en distingue aussi à d'autres égards. L’identification des points de rapprochement et de divergence permet de spécifier la critique formulée par Robert Salais.

Maurice Halbwachs était favorable à l'utilisation de la recherche statistique en sociologie ${ }^{21}$, dans laquelle il voyait « le seul moyen de reconnaître les régularités sociales ${ }^{22}$, les caractères

${ }^{19} \mathrm{R}$. SALAIS, « La donnée n'est pas un donné. Pour une analyse critique de l'évaluation chiffrée de la performance », loc. cit., pp. 514-515.

${ }^{20}$ James C. ScOTT, Seeing like a State: How Certain Schemes to Improve the Human Condition Have Failed, New Haven, Yale University Press, 1998.

${ }^{21}$ Pour une présentation générale, v. O. MARTIN, "Raison statistique et raison sociologique chez Maurice Halbwachs », Revue d'histoire des sciences humaines, n 1, 1999, pp. 69-101. Pour une mise en perspective : Z. BAYATRIZI et Th. KEMPLE, «Un problème de chiffres : l'utilisation des connaissances empiriques en statistique dans la théorie sociale classique ", Sociologie et sociétés, vol. 44, $\mathrm{n}^{\circ}$ 2, 2012, p. 45-73.

${ }^{22} \mathrm{M}$. HALBWACHS, «La statistique en sociologie ", in La statistique. Ses applications, les problèmes qu'elle soulève, Paris, PUF, 1935, p. 114. Henri LEVY-BRUHL reprend ce raisonnement à propos des régularités juridiques : « notre connaissance des faits juridiques ne saurait, pas plus que celle des faits économiques, se passer de données numériques précises et méthodiquement établies » (dans le même volume, H. LEVY-BRUHL, " Note sur la statistique et le droit», pp. 141-147). Mais au-delà de la connaissance qu'elles permettent, les statistiques offrent aussi des ressources pour l'action: "une société rationnelle se 
collectifs au-delà des destins individuels ${ }^{23}$. Il rejoint également François Simiand pour considérer la statistique comme « une certaine sorte de recherche expérimentale ${ }^{24}$, susceptible d'éclairer non seulement les faits de la nature mais aussi les faits sociaux. Mais Halbwachs est aussi très sensible aux limites que présente l'outil statistique ${ }^{25}$. Si les statistiques permettent bel et bien de faire ressortir des régularités masquées par la dispersion des situations individuelles, elles ne disent rien de leurs causes. Une analyse sociologique est indispensable pour en saisir véritablement la signification. Ainsi, à propos des causes du suicide : " les statistiques nous indiquent combien il y a eu de suicides dans un groupe. Mais elles ne nous font pas connaître à quel ordre de faits sociaux, domestiques, religieux, politiques, économiques chaque catégorie d'entre eux se rattachent $»^{26}$. De même, le résultat statistique dépend nécessairement des intentions de ceux qui conçoivent l'enquête et ses instruments. D’où cette mise en garde de Maurice Halbwachs : « il ne faut demander à une expression statistique que ce qu'elle peut nous donner, que ce que veulent atteindre ceux qui la calculent $»^{27}$, et toujours rester attentif à ce qui reste en dehors du champ de vision.

Sur ce point, la critique de Robert Salais semble s'inscrire dans la continuité des travaux de Maurice Halbwachs lorsqu'il dénonce le fait que les statistiques deviennent des fins en soi $^{28}$. Pour l'un, comme pour l'autre, « c'est la logique du social, de l'empirie, qui doit primer sur la logique des mathématiques, la logique des statistiques (...) $»^{29}$. En aucun cas, le recours à la gouvernerait essentiellement à l'aide de statistiques » (p. 143).

${ }^{23} \ll(. .$.$) la statistique permet d'atteindre les caractères d'un groupe, qui ont une réalité pour le$ groupe tout entier, mais qu'on ne découvrirait dans aucun membre de ce groupe pris à part et isolément » (M. HALBWACHS, "La statistique en sociologie », loc. cit., p. 114). Sur cette démarche, on se reportera également à M. HALBWACHS, La théorie de l'homme moyen. Essai sur Quételet et la statistique morale, Paris, Librairie Félix Alcan, 1913 ; M. HALBWACHS, L'évolution des besoins dans les classes ouvrières, Paris, Librairie Félix Alcan, 1933.

${ }^{24} \mathrm{~F}$. SIMIAND, Statistique et Expérience. Remarques de méthode, Paris, Librairie des sciences politiques et sociales, 1922, spéc. pp. 18-19. Sur les liaisons entre expérimentation en physique et en sociologie, et sur l'influence exercée ici par Émile Durkheim, v. plus particulièrement Ch. TOPALOV, «'Expériences sociologiques' : les faits et les preuves dans les thèses de Maurice Halbwachs (1909-1913) ", Revue d'histoire des sciences humaines, $\mathrm{n}^{\circ} 1$, 1999, spéc. pp. 20 et s.

${ }^{25}$ Pour un exposé détaillé, voir O. MARTIN, «Raison statistique et raison sociologique chez Maurice Halbwachs », loc. cit., spéc. pp. 76 et s.

${ }^{26}$ M. HalbWACHS, Les causes du suicide, Paris, PUF, coll. " Le lien social », [1930] 2002, pp. 368-369.

${ }^{27} \mathrm{M}$. HALBWACHS, L'évolution des besoins dans les classes ouvrières, op. cit., p. 2.

${ }^{28} \mathrm{R}$. SALAIS, « La donnée n'est pas un donné. Pour une analyse critique de l'évaluation chiffrée de la performance », loc. cit., spéc. p. 506 et s.

${ }^{29} \mathrm{O}$. MARTIN, « Raison statistique et raison sociologique chez Maurice Halbwachs », loc. cit., p. 81. 
formalisation ne doit se payer d'un défaut d'attention au social. Et cela est vrai, pour Maurice Halbwachs comme pour Robert Salais, qu'elle soit le fait de statisticiens ou d'économistes ${ }^{30}$. L'intervention de Robert Salais ne met donc pas tant en cause le principe du recours aux statistiques, qui est déjà ancien dans les sciences sociales, que la manière dont sont articulés la formalisation et les faits sociaux, la logique mathématique et l'empirie. Les usages des statistiques dénoncés par Robert Salais manifestent, en effet, une inversion de cette hiérarchie, une victoire de la formalisation et de la modélisation sur l'empirie, alors même qu'Halbwachs avait montré leur faible pouvoir explicatif.

Au-delà de ce rapprochement, une différence mérite cependant d’être soulignée. Car, en effet, la critique de Robert Salais ne porte pas d'abord sur l'incapacité de la statistique à décrire correctement le social, mais bien plutôt sur le fait qu'elle est « difficilement compatible avec la justice sociale », qu'elle écarte l'action publique « des impératifs de la démocratie » ${ }^{31}$. Cette dernière observation nous ramène à une interrogation déjà rencontrée précédemment ${ }^{32}$ : comment mettre en place un appareil statistique et des indicateurs qui ne fassent pas prévaloir des valeurs qui n’auraient pas été délibérées démocratiquement ? Ce questionnement est évidemment crucial dès lors que l’on reconnaît la normativité inhérente aux dispositifs techniques de recueil des informations. La concrétisation de réponses opérationnelles reste un chantier largement ouvert.

\footnotetext{
${ }^{30}$ Halbwachs critique fortement l'inclinaison de l'économie politique de son temps vers une trop grande formalisation (v. Ch. TopALOV, «'Expériences sociologiques' : les faits et les preuves dans les thèses de Maurice Halbwachs (1909-1913) », loc. cit., p. 19 et s.). Robert Salais rejoint cette critique dans ses propres travaux (v. par ex. R. SALAIS, E. CHATEL et D. RiVAUD-DANSET (dir.), Institutions et conventions. La réflexivité de l'action économique, Paris, Editions de l'EHESS, coll. « Raisons Pratiques », 1999).

${ }^{31} \mathrm{R}$. SALAIS, « La donnée n'est pas un donné. Pour une analyse critique de l'évaluation chiffrée de la performance », loc. cit., ; R. SALAIS, «Indicateurs et conduite des politiques publiques : où sont les problèmes ? ", loc. cit.

${ }^{32}$ Cf. supra, 1 . c.
} 